\title{
Atuação do enfermeiro no manejo da crise em saúde mental: Uma revisão integrativa
}

\author{
Nurses' performance in the management of the mental health crisis: An integrative review \\ Desempeño de las enfermeras en la gestión de la crisis de salud mental: Una revisión integradora
}

Recebido: 08/07/2021 | Revisado: 16/07/2021 | Aceito: 19/07/2021 | Publicado: 26/07/2021

\author{
Anny Caroline Santos Almeida \\ ORCID: https://orcid.org/0000-0002-8471-4750 \\ Universidade Federal de Sergipe, Brasil \\ E-mail: annycaroline.ufs@gmail.com \\ Carla Kalline Alves Cartaxo Freitas \\ ORCID: https://orcid.org/0000-0001-7604-9132 \\ Universidade Federal de Sergipe, Brasil \\ E-mail: carlakalline@gmail.com \\ Andreia Freire de Menezes \\ ORCID: https://orcid.org/0000-0002-2972-8236 \\ Universidade Federal de Sergipe, Brasil \\ E-mail: deiamenezes1@ @otmail.com \\ Monaline Santos Almeida \\ ORCID: https://orcid.org/0000-0002-5726-3085 \\ Universidade Federal de Sergipe, Brasil \\ E-mail: Monalineonline@ academico.ufs.br \\ Anna Paula Santos Almeida \\ ORCID: https://orcid.org/0000-0001-5929-7628 \\ Universidade Federal de Sergipe, Brasil \\ E-mail: annapaulaufs@gmail.com
}

\begin{abstract}
Resumo
A crise em saúde mental trata-se de uma condição complexa, que necessita de uma assistência eficiente e humanizada, concebida como uma expressão que envolve a capacidade subjetiva do sujeito em responder a situações desencadeantes em qualquer momento da vida. Esse estudo teve como objetivo consolidar evidências científicas que discutam a respeito da assistência de enfermagem ofertada no atendimento à pessoa em crise psíquica. Trata-se de um estudo bibliográfico, tipo revisão integrativa, a partir de estudos primários, descritivos, exploratórios e de abordagem qualitativa e quantitativa, por meio da análise de artigos científicos e teses, disponíveis em base de dados eletrônicos de acesso livre. Os artigos selecionados para a composição da amostra foram codificados, para então, prosseguirmos com a sintetização dos resultados, agrupados em duas categorias principais, a saber: Lógica biomédica dos fenômenos psíquicos (práticas mecanicistas) e produção de vínculo, autonomia e a corresponsabilização como estratégia de cuidado. A atuação da enfermagem é permeada por limites e desafios, resultantes de um processo de trabalho conturbado e as evidências científicas no atendimento à crise psíquica reafirmam a permanência do modelo biomédico hegemônico, abordando assim de maneira reducionista os demais aspectos primordiais da assistência, pautados pela Reforma Psiquiátrica. Percebe-se, com este estudo, o quanto é valioso discutir sobre crise, produção de singularidades e principalmente, sobre as práticas de enfermagem na atenção à pessoa na crise.
\end{abstract}

Palavras-chave: Intervenção na crise; Assistência à saúde mental; Cuidados de enfermagem.

\begin{abstract}
The mental health crisis is a complex condition that requires efficient and humanized care, conceived as an expression that involves the subject's subjective ability to respond to triggering situations at any time in life. This study aimed to consolidate scientific evidence that discusses the nursing care offered in the care of people in psychological crisis. This is a bibliographic study, integrative review type, based on primary, descriptive, exploratory studies with a qualitative and quantitative approach, through the analysis of scientific articles and theses, available in an open access electronic database. Consolidated on the premise of the six phases of the integrative review elaboration process. The articles selected for the composition of the sample were coded, and then we proceed with the synthesis of the results, grouped into two main categories, namely: Biomedical logic of psychic phenomena (mechanistic practices) and production of bond, autonomy and co-responsibility as a strategy of care. The role of nursing is permeated by limits and challenges, resulting from a troubled work process and scientific evidence in the care of the psychic crisis reaffirms the permanence of the hegemonic biomedical model, thus approaching in a reductionist way the other essential aspects of care, guided by the Reform Psychiatric. It is clear, with this study, how valuable it is to discuss about crisis, production of singularities and, above all, about nursing practices in the care of people in crisis.
\end{abstract}

Keywords: Crisis intervention; Mental health assistance; Nursing care. 


\begin{abstract}
Resumen
La crisis de salud mental es una condición compleja que requiere de un cuidado eficiente y humanizado, concebido como una expresión que involucra la capacidad subjetiva del sujeto para responder a situaciones desencadenantes en cualquier momento de la vida. Este estudio tuvo como objetivo consolidar la evidencia científica que discute los cuidados de enfermería ofrecidos en el cuidado de personas en crisis psicológica. Se trata de un estudio bibliográfico, tipo revisión integradora, basado en estudios primarios, descriptivos, exploratorios con enfoque cualitativo y cuantitativo, mediante el análisis de artículos y tesis científicas, disponibles en una base de datos electrónica de acceso abierto. Consolidado sobre la premisa de las seis fases del proceso de elaboración de la revisión integradora. Se codificaron los artículos seleccionados para la composición de la muestra, para luego proceder a la síntesis de los resultados, agrupados en dos categorías principales, a saber: Lógica biomédica de fenómenos psíquicos (prácticas mecanicistas) y producción de vínculo, autonomía y corresponsabilidad como estrategia de atención. El rol de la enfermería está impregnado de límites y desafíos, producto de un proceso de trabajo convulso y la evidencia científica en el cuidado de la crisis psíquica reafirma la permanencia del modelo biomédico hegemónico, acercándose así de manera reduccionista a los demás aspectos esenciales del cuidado, guiados por la Reforma Psiquiátrica. Queda claro, con este estudio, lo valioso que es discutir sobre crisis, producción de singularidades y, sobre todo, sobre prácticas de enfermería en el cuidado de personas en crisis.
\end{abstract}

Palabras clave: Intervención en la crisis; Atención a la salud mental; Atención de enfermería.

\title{
1. Introdução
}

A loucura e suas representações sociais vêm sendo construídas socioeconomicamente desde primórdios históricos (Amarante, 2007). No entanto, discussões sobre o modelo biomédico, a violação dos direitos humanos e de cidadania fizeram emergir o movimento de reforma psiquiátrica, que trouxe uma nova forma de pensar sobre o processo saúde-doença por meio da valorização do cuidado ao sujeito com base no território e em suas vivências (Basaglia, 1979; Silva et al., 2020).

No modelo clássico da psiquiatria, a crise é entendida como uma situação na qual há grave disfunção psíquica ou comportamental (Willrich et al., 2014). Caracterizada por atos de violência que podem envolver agressividade verbal e física, ideações suicidas, tentativas de suicídio, crises depressivas, alucinação, excitação maníaca, automutilação, autonegligência, juízo crítico e surtos psicóticos (Veloso et al., 2018), ocorrendo exclusivamente em decorrência da doença de base psicológica preexistente (Amarante, 2007).

Já no contexto reformista, a crise é concebida como uma expressão que envolve a capacidade subjetiva do sujeito em responder a situações desencadeantes em qualquer momento da vida (Basaglia, 1979). Nessa perspectiva, reconhece o outro como legítimo cidadão de direitos e valoriza os diferentes sujeitos, implicados na produção de sua saúde (Portal et al.,2021; Yasui, 2013).

Uma das demandas que mais gera dificuldades nesse contexto de mudanças paradigmáticas é a atenção à crise, considerada um dos aspectos mais difíceis e estratégicos no processo da Reforma Psiquiátrica (Amarante, 2007; Silva et al., 2020). Sendo considerada uma emergência psiquiátrica, pois a pessoa não consegue resolver o problema com seus mecanismos habituais de enfrentamento. O primeiro atendimento muitas vezes é dado no pronto-socorro, assim como a estabilização da crise e a introdução do tratamento (Del-Ben et al., 1999). Independentemente da denominação que recebe, trata-se de uma condição complexa, em que o sofrimento muitas vezes vem acompanhado não somente de problemas emocionais ou psicológicos, mas que necessita de uma assistência rápida e adequada (Veloso et al., 2018).

Os serviços de assistência substitutivos do modelo manicomial no atendimento à crise são prestados pelos componentes integrados da Rede de atenção Psicossocial (RAPS): Centros de Atenção Psicossocial (CAPS) nas suas diferentes modalidades, emergências dos hospitais gerais, atenção à urgência/emergência nos prontos socorros, atenção primária à saúde por meio das Unidades Básicas de Saúde (UBS) e, os Serviços de Atendimento Móvel de Urgência (SAMU) (Brasil, 2011; Portal et al.,2021).

Para o Ministério da Saúde do Brasil, não há local específico e, exclusivamente responsável, para atender às situações de crise, nem sendo tampouco de exclusividade dos profissionais de saúde. $\mathrm{O}$ atendimento à crise deve acontecer no lugar onde 
se encontra o indivíduo, ou seja, no seu ambiente de vida ou de cuidado (Brasil, 2013). Sabe-se que a equipe de enfermagem representa em termos numéricos, a maior força de trabalho das instituições componentes da RAPS e a sua atuação, vai além das atividades assistenciais e repercutem na dinâmica com o usuário e com familiares (Oliveira, Garcia \& Toledo, 2017).

Entendendo que a importância deste tema para a enfermagem reside no fato de que, ao desvendar como as intervenções se consolidam, poderemos abrir caminhos para ressignificação de conceitos e propostas aos atendimentos a diferentes manifestações proporcionadas pelo fenômeno da loucura, como no momento de crise (Oliveira et al., 2020).

E a partir dessa primeira análise formulou-se a seguinte questão norteadora: Quais as evidências presentes na literatura científica sobre a assistência de enfermagem ofertada à pessoa em crise psiquiátrica? Diante do exposto, tem-se por objetivo a consolidação de evidências literárias que discutam a respeito da assistência de enfermagem ofertada no atendimento à crise psíquica.

\section{Metodologia}

Trata-se de um estudo bibliográfico do tipo revisão integrativa, realizado a partir da seguinte questão norteadora: Quais as evidências presentes na literatura científica sobre a assistência de enfermagem ofertada à pessoa em crise psiquiátrica? Foi utilizado o método de Cooper com as seguintes etapas: formulação do problema, coleta dos dados, avaliação dos dados, análise e interpretação e apresentação pública (Mendes et al., 2008).

Os artigos científicos foram selecionados através de uma busca bibliográfica nas bases de dados de Literatura LatinoAmericana e do Caribe em Ciências da Saúde (LILACS), Medical Literature Analysis and Retrieval System Online (MEDLINE), US National Library of Medicine National Institutes of Health (PubMed) e nas bibliotecas eletrônicas Scientific Eletronic Library Online (SCIELO), Biblioteca Virtual em Saúde (BVS) e Base de Dados de Enfermagem (BDENF). Com a utilização dos descritores contolados "Intervenção na Crise, Assistência à Saúde Mental, Cuidados de Enfermagem, Enfermagem Psiquiátrica", unidos pelo operador booleano AND. O levantamento bibliográfico ocorreu entre novembro de 2020 a junho de 2021.

Estabeleceram-se como critérios de inclusão, manuscritos publicados de 2010 à 2020, cujo texto completo estivesse disponível no idioma português, realizados no Brasil, e que abordassem a assistência e/ou a atuação de Enfermagem e/ou o cuidado de Enfermagem no enfrentamento da crise em saúde mental. Excluíram-se os estudos que não abordavam a temática proposta pela revisão, artigos duplicados e os não disponibilizados na integra online.

Foram encontrados 102 artigos dos quais 86 tinham o texto completo disponível, 9 foram excluídos por duplicidade, totalizando 77. Inicialmente 42 artigos foram selecionados por título e, em seguida, 31 pela leitura do resumo. Ao final, foram selecionados 10 artigos pela leitura do texto completo (Figura 1). O processo metodológico de refinamento dos artigos realizou-se a partir da leitura em pares, em dupla avaliação. 
Research, Society and Development, v. 10, n. 9, e31010918030, 2021

(CC BY 4.0) | ISSN 2525-3409 | DOI: http://dx.doi.org/10.33448/rsd-v10i9.18030

Figura 1. Fluxograma de identificação e seleção dos artigos. Aracaju, SE, Brasil, 2021.

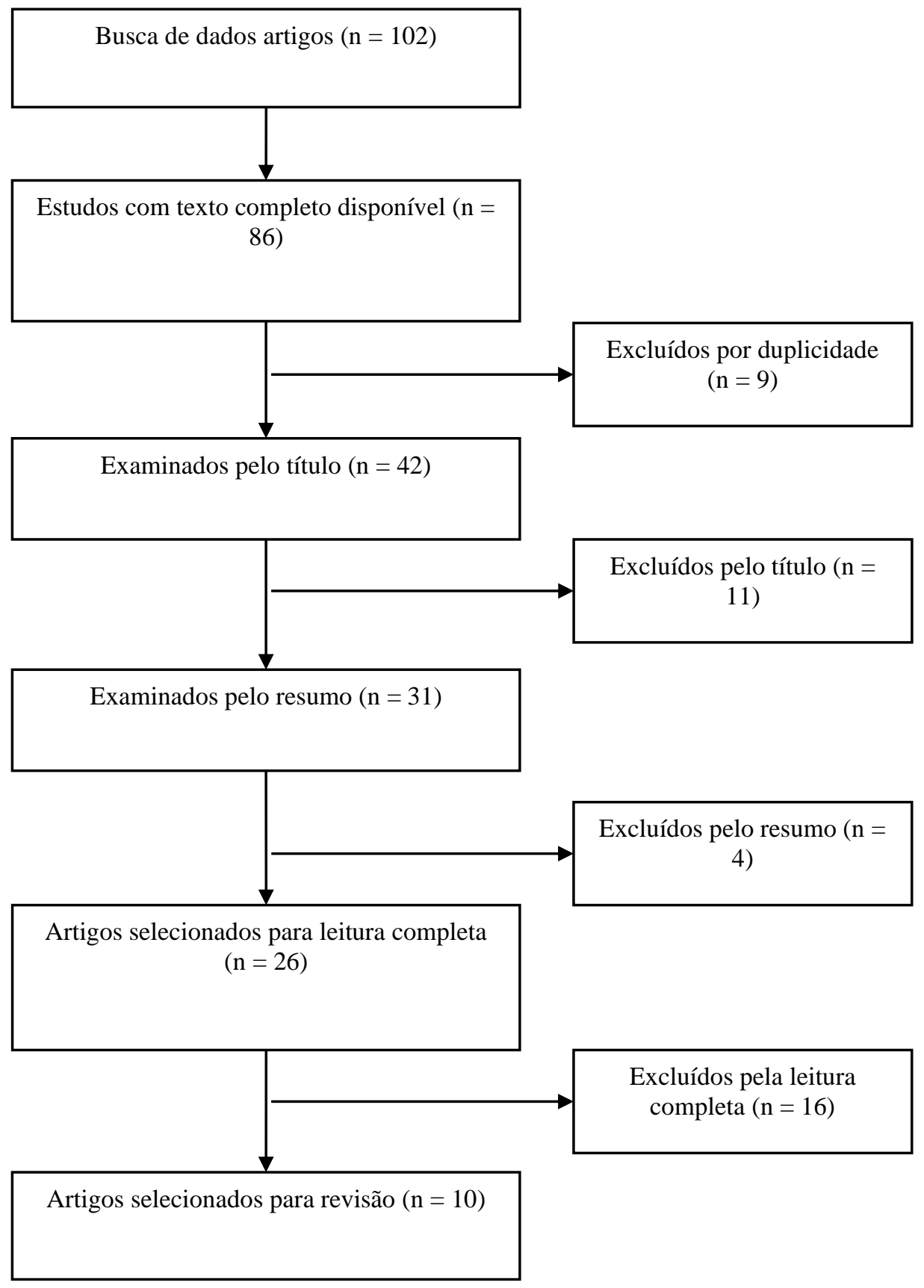

Fonte: Autores.

\section{Resultados}

Os artigos incluídos na análise final estão apresentados no Tabela 1. Em seguida, foi realizada a análise crítica dos resultados através da síntese descritiva dos estudos. Observou-se uma escassez de publicações referentes as intervenções de enfermagem nos serviços de atendimento frente às crises psiquiátricas. 
Tabela 1 - Caracterização dos artigos selecionados para composição da amostra. Aracaju, SE, Brasil, 2021.

\begin{tabular}{|c|c|c|c|}
\hline Autores & Tipo de Pesquisa & Objetivos & Amostra \\
\hline Oliveira et al. & $\begin{array}{l}\text { Estudo descritivo de natureza } \\
\text { qualitativa. }\end{array}$ & $\begin{array}{l}\text { Compreender como os trabalhadores de enfermagem } \\
\text { percebem o cuidado às pessoas em situações de } \\
\text { urgência e emergência psiquiátricas no Serviço de } \\
\text { Atendimento Móvel de Urgência (SAMU). }\end{array}$ & $\begin{array}{lr}\text { Trinta e } & \text { quatro } \\
\text { profissionais } & \text { de } \\
\text { enfermagem. } & \end{array}$ \\
\hline Silva et al & Estudo qualitativo. & $\begin{array}{l}\text { Analisar as características do Acolhimento Noturno } \\
\text { em um Centro de Atenção Psicossocial III. }\end{array}$ & $\begin{array}{l}\text { Quinze profissionais de } \\
\text { enfermagem. }\end{array}$ \\
\hline Pereira et al & $\begin{array}{l}\text { Estudo qualitativo, descritivo } \\
\text { e exploratório. }\end{array}$ & $\begin{array}{l}\text { Analisar as dificuldades encontradas pelos } \\
\text { enfermeiros no cuidado a pessoa com comorbidade } \\
\text { psiquiátrica. }\end{array}$ & Doze enfermeiros. \\
\hline Veloso et al & Estudo retrospectivo. & $\begin{array}{l}\text { Analisar os atendimentos de natureza psiquiátrica } \\
\text { realizados pelo serviço pré-hospitalar móvel de } \\
\text { urgência. }\end{array}$ & $\begin{array}{l}\text { Mil e oitenta e oito } \\
\text { atendimentos. }\end{array}$ \\
\hline $\begin{array}{l}\text { Oliveira; Garcia; } \\
\text { Toledo }\end{array}$ & $\begin{array}{l}\text { Estudo qualitativo de } \\
\text { abordagem fenomenográfica. }\end{array}$ & $\begin{array}{l}\text { Conhecer como o enfermeiro realiza o cuidado de } \\
\text { enfermagem ao paciente em primeiro surto psicótico. }\end{array}$ & Dez enfermeiros. \\
\hline Buriola et al & Estudo qualitativo. & $\begin{array}{l}\text { Apreender a percepção de profissionais, usuários e } \\
\text { familiares acerca da atuação do enfermeiro. }\end{array}$ & $\begin{array}{l}\text { Quinze profissionais, } \\
\text { nove usuários e seis } \\
\text { familiares. }\end{array}$ \\
\hline Zeferino et al & $\begin{array}{lr}\text { Pesquisa } & \text { exploratória } \\
\text { descritiva com } & \text { abordagem } \\
\text { qualitativa. } & \end{array}$ & $\begin{array}{l}\text { Conhecer } \\
\text { o cuidado prestado às pessoas } \\
\text { em situação } \\
\text { de crise em serviços de saúde mental. }\end{array}$ & $\begin{array}{l}\text { Quatrocentos e vinte e } \\
\text { nove alunos da primeira } \\
\text { edição do Curso Crise e } \\
\text { Urgência em Saúde } \\
\text { Mental e trabalhadores. }\end{array}$ \\
\hline Willrich et al & Pesquisa qualitativa. & $\begin{array}{l}\text { Conhecer as práticas de profissionais produzidos pelas } \\
\text { novas formas de lidar e se relacionar com o sujeito em } \\
\text { crise psíquica grave. }\end{array}$ & $\begin{array}{l}\text { Vinte e sete } \\
\text { profissionais. }\end{array}$ \\
\hline Willrich et al & Estudo qualitativo. & $\begin{array}{l}\text { Identificar as ações de atenção à crise no território e } \\
\text { os sentidos que as envolvem. }\end{array}$ & $\begin{array}{l}\text { Vinte e sete } \\
\text { profissionais } \\
\text { e três diários de campo }\end{array}$ \\
\hline & & & $\begin{array}{l}\text { com registro de } \\
\text { trezentos e noventa } \\
\text { horas de observação. }\end{array}$ \\
\hline Borges et al & Estudo qualitativo & $\begin{array}{l}\text { Conhecer a visão da equipe de enfermagem sobre o } \\
\text { atendimento à crise psíquica em uma } \\
\text { unidade de emergência de um Pronto-Socorro. }\end{array}$ & $\begin{array}{l}\text { Dois enfermeiros e três } \\
\text { técnicos de } \\
\text { Enfermagem }\end{array}$ \\
\hline
\end{tabular}

Fonte: Autores (2021).

Procedeu-se a extração das informações dos estudos selecionados, para que uma reavaliação da revisão pudesse ocorrer de forma mais apurada. Os artigos escolhidos para a composição da amostra foram codificados, para então, prosseguirmos com a sintetização dos resultados. Tais códigos são representados pela letra "A", seguida do número correspondente a um dos artigos, exemplo: A1, A2, A3... 
Os resultados foram agrupados em duas categorias empíricas, como demonstra o Tabela 2, a saber: Lógica biomédica dos fenômenos psíquicos (práticas mecanicistas) e produção de vínculo, autonomia e a corresponsabilização como estratégia de cuidado.

Tabela 2 - Categorização dos artigos selecionados e análise de conteúdo. Aracaju, SE, Brasil, 2021.

\begin{tabular}{|c|c|c|c|}
\hline Categorias & Código & Autores & Ano de publicação \\
\hline \multirow[t]{6}{*}{$\begin{array}{l}\text { Lógica biomédica dos fenômenos } \\
\text { psíquicos (práticas mecanicistas). }\end{array}$} & A1 & Oliveira et al & 2020 \\
\hline & A3 & Pereira et al & 2019 \\
\hline & A4 & Veloso et al & 2018 \\
\hline & A5 & Oliveira, Garcia \& Toledo & 2017 \\
\hline & A8 & Willrich et al & 2014 \\
\hline & A10 & Borges et al & 2012 \\
\hline \multirow{4}{*}{$\begin{array}{l}\text { Produção de vínculo, autonomia e } \\
\text { a corresponsabilização como } \\
\text { estratégia de cuidado. }\end{array}$} & A2 & Silva et al & 2020 \\
\hline & A6 & Buriola et al & 2016 \\
\hline & A7 & Zeferino et al. & 2016 \\
\hline & A9 & Willrich et al & 2013 \\
\hline
\end{tabular}

Fonte: Autores (2021).

\section{Discussão}

\subsection{Lógica biomédica dos fenômenos psíquicos (práticas mecanicistas)}

Evidenciou-se a recorrência das discussões pautadas no modelo biomédico que por décadas foi hegemônico entre os modelos de atenção à saúde (Oliveira, Garcia \& Toledo, 2017; Pereira et al., 2019; Veloso et al., 2018; Willrich et al., 2014; Borges et al., 2012). A sociedade desenvolveu um comportamento segregacionista, excludente e que estimulava a rotulagem da crise e daqueles que estão em sofrimento mental (Amarante, 2007).

Certamente, esse preconceito com a loucura não é uma característica exclusiva da equipe de enfermagem ou demais profissionais que prestam assistência direta no contexto psiquiátrico, mas da sociedade em que estamos inseridos (Veloso et al., 2018). Fato que é agravado pelo pouco conhecimento da atuação do enfermeiro dentro de um sistema de saúde complexo, consolidando assim práticas pouco reflexivas e reprodutivas do modelo biomédico (Oliveira et al., 2020; Veloso et al., 2018; Willrich et al., 2014). A crise é o momento em que a sociedade enxerga o transtorno mental nesse contexto conflituoso ao indivíduo e que por vezes em caso de agressividade na qual o usuário possa criar confusão pela rua, assustar, ameaçar as pessoas e então cria-se uma situação de desordem pública (Willrich et al., 2014).

Os serviços de urgência ocupam um lugar estratégico e ao mesmo tempo problemático na RAPS, pois uma das condutas nos casos após o atendimento emergencial o principal direcionamento é a repressão da crise (Buriola et al., 2016; Oliveira et al., 2020). A conduta adotada é silenciar a loucura, isto é, como forma de intervenção no contexto singular da crise, 
o maior percentual dos estudos analisados traz medidas como a contenção medicamentosa, contenção mecânica e a internação psiquiátrica (Oliveira et al., 2020; Pereira, Duarte \& Eslabao, 2019; Veloso et al., 2018; Oliveira, Garcia \& Toledo, 2017; Willrich et al., 2014; Borges et al., 2012).

Ações que se limitam à diminuição de sintomas, sem espaço e tempo para que o sujeito manifeste seu sofrimento e exteriorize sua singularidade (Amarante, 2007; Zeferino et al., 2016). Sendo assim, práticas divergentes ao preconizado na Reforma Psiquiátrica Brasileira e compactuantes com o modelo manicomial (Veloso et al., 2018).

Notavelmente, torna-se um paradoxo na assistência que muitas vezes é refletido na fragilidade da estrutura organizacional das unidades de saúde mental, em função da sobrecarga de atividades, inadequação do espaço físico, insegurança e falta de preparo, aliado ao baixo número de funcionários disponíveis para prestar o atendimento, consolidando uma prática qualificada (Pereira, Duarte \& Eslabao, 2019; Oliveira, Garcia \& Toledo, 2017; Willrich et al., 2014).

Os avanços nas práticas de saúde mental, nas últimas décadas, são inegáveis, mas ainda há muitos desafios a serem superados e ofertas de cuidados a serem repensados (Del-Ben et al., 1999; Willrich et al., 2013). Principalmente quanto ao lugar que ocupa o uso das medicações nas práticas de cuidado, em especial, quando o foco é a atenção às crises de forma hegemônica (Oliveira et al., 2020; Oliveira, Garcia \& Toledo, 2017; Veloso et al., 2018).

Tal realidade contraria a lógica da Rede de Atenção Psicossocial e seus dispositivos integrantes, visto que esta privilegia o atendimento em hospitais gerais ou em outros serviços extra-hospitalares de saúde mental de base comunitária, mencionando o CAPS como instituição de referência no território para o manejo das crises (Amarante, 2007).

\subsection{Produção de vínculo, autonomia e a corresponsabilização como estratégia de cuidado}

A humanização do atendimento em saúde mental possibilita a melhoria da qualidade da atenção prestada, propiciando, consequentemente, uma nova experiência entre profissionais e pacientes, gerando novas práticas cuidadoras (Silva et al., 2020; Buriola et al., 2016; Zeferino et al., 2016; Willrich et al., 2013). O ato de humanizar o atendimento significa dar o devido valor ao outro, reconhecer a sua integralidade, seu sofrimento e suas necessidades (Amarante,2007), acolhendo as angústias, as dores e o sofrimento diante da fragilidade do corpo e da mente (Willrich et al., 2014).

Neste sentido, não é a categoria profissional da enfermagem o fator relevante que determinará o atendimento direto à crise, mas sim o vínculo que a equipe possui com o sujeito (Borges et al., 2012), caracterizando assim a prática na Atenção Psicossocial como multiprofissional e interdisciplinar (Willrich et al., 2014).

Os sujeitos em crise precisam de profissionais atentos e disponíveis que estejam juntos e entendam o contexto da crise, sendo um ponto de apoio, um laço de afeto e de respeito que ajude nessa experiência conflitante (Silva et al., 2020; Buriola et al., 2016; Zeferino et al., 2016; Willrich et al., 2013). Assim, surge a necessidade da reorganização da prática e a reformulação das atribuições dos profissionais de enfermagem na prestação do cuidado em saúde mental, uma vez que a enfermagem nasceu junto com o manicômio e teve sua trajetória marcada pela responsabilidade em manter a ordem interna da instituição (Del-Ben et al., 1999; Willrich et al., 2014).

Inserindo-se no contexto de clínica ampliada, na qual são utilizados diversos saberes e recursos, com isso estão sendo construídos novas abordagens e instrumentos para o cuidado pautado na ética e singularidade do sujeito (Amarante, 2007). Nesse contexto, é possível reconhecer que o vínculo entre a equipe de enfermagem e usuários é o ponto inicial para o processo terapêutico adequado e ganha novas perspectivas.

Com as mudanças preconizadas pela Reforma Psiquiátrica e com a inserção das residências multiprofissionais em saúde (Buriola et al., 2016; Zeferino et al., 2016; Willrich et al., 2013), utilizando de recursos terapêuticos humanísticos, a citar, as noções de escuta terapêutica, o acolhimento, os planos terapêuticos individualizados e as práticas coletivas (Silva et al., 2020; Buriola et al., 2016; Willrich et al., 2013). 


\section{Conclusão}

De acordo com os achados, a atuação da enfermagem é permeada por limites e desafios, resultantes de um processo de trabalho conturbado, onde o mesmo se insere. Contudo sua presença no manejo a crise psiquiátrica é fundamental à dinâmica de trabalho da equipe de saúde mental.

As evidências científicas a respeito da assistência de enfermagem ofertada no atendimento à crise psíquica reafirmam a permanência do modelo biomédico hegemônico nas práticas de cuidado em saúde e na compreensão dos fenômenos psíquicos. O conhecimento da equipe está relacionado a um atendimento voltado para as questões clínicas. Para isso, os profissionais se utilizam das contenções como instrumentos de abordagem a comportamentos considerados inadequados ou irrelevantes. O cuidado ocorre de maneira fragmentada, principalmente devido ao conhecimento teórico insuficiente e falta de confiança e habilidades de interação interpessoal em equipe.

Abordando assim de maneira reducionista os demais aspectos primordiais da assistência, pautados pela Reforma Psiquiátrica, como a produção de vínculo, a autonomia e a corresponsabilização nos processos de saúde. Além disso, espera-se que este estudo possa contribuir com a prática profissional da equipe de enfermagem ao demonstrar a necessidade de discussão dos processos de qualificação da equipe que atua no manejo a crise e sobre as novas abordagens em saúde mental.

\section{Referências}

Amarante, P. (2007). Saúde mental e atenção psicossocial. Fiocruz.

Basaglia, F. (1979). A Psiquiatria Alternativa: contra o pessimismo da razão o otimismo da prática. Brasil Debates.

Borges, L. da R., Pinho, L. B. de, Lacchini, A. J. B., \& Schneider, J. F. (2012). Atendimento à crise psíquica no pronto-socorro: visão de profissionais de enfermagem. Revista Gaúcha de Enfermagem, 33(3), 27-33. https://doi.org/10.1590/s1983-14472012000300004

Brasil. (2011). Portaria $N^{o} 3.088$, de 23 de dezembro de 2011. Institui a Rede de Atenção Psicossocial para pessoas com sofrimento ou transtorno mental e com necessidades decorrentes do uso de crack, álcool e outras drogas, no âmbito do Sistema Único de Saúde. Brasília, DF: Ministério da Saúde. https://bvsms.saude.gov.br/bvs/saudelegis/gm/2011/prt3088_23_12_2011_rep.html

Brasil. (2013). Cadernos de Atenção Básica, $n^{o}$ 34. Brasília, DF: Ministério da Saúde. https://bvsms.saude.gov.br/bvs/publicac oes/cadernos_atencao_basica_34_saude_mental.pdf.

Buriola, A. A., Kantorski, L. P., Sales, C. A., \& Matsuda, L. M. (2016). Nursing Practice At a Psychiatric Emergency Service: Evaluation Using Fourth Generation Assessment. Texto \& Contexto - Enfermagem, 25(1), 1-9. https://doi.org/10.1590/0104-070720160004540014

Corrêa, L., Pinto Valério, M., Oliveira Teixeira, A., Guerreiro, L. F., Fossati Silveira, D., Terra Machado, P., Botelho Xavier, B. E., Oliz, M. M., Silveira Heredia Y Antunes, D., \& Goularte Knuth, A. (2014). A atuação da educação física nas residências multiprofissionais em saúde. Revista Brasileira Em Promoção Da Saúde, 27(3), 428-433. https://doi.org/10.5020/18061230.2014.p4284

Del-Ben, C. M., Marques, J. M. A., Jr, A. S., \& Zuardi, A. W. (1999). Políticas de saúde mental e mudanças na demanda de serviços de emergência. Revista Saúde Pública, 33(5). https://doi.org/10.1590/S0034-89101999000500006

Mendes, K. D. S., Silveira, R. C. de C. P., \& Galvão, C. M. (2008). Revisão integrativa: método de pesquisa para a incorporação de evidências na saúde e na enfermagem. Texto \& Contexto - Enfermagem, 17(4), 758-764. https://doi.org/10.1590/s0104-07072008000400018

Oliveira, A. de, Garcia, A. P. R. F., \& Toledo, V. P. (2017). Patterns of knowledge used by nurses in caring for the patient in the first psychotic outbreak. Escola Anna Nery, 21(3), 1-8. https://doi.org/10.1590/2177-9465-ean-2017-0001

Oliveira, L. C. de, Menezes, H. F. de, Oliveira, R. L. de, Lima, D. M. de, Fernandes, S. F., \& Silva, R. A. R. da. (2020). Mobile care service for psychiatric urgencies and emergencies: perception of nursing workers. Revista Brasileira de Enfermagem, 73(1), e20180214. https://doi.org/10.1590/0034-7167-20180214

Pereira, L. P., Duarte, M. de L. C., \& Eslabão, A. D. (2019). O cuidado à pessoa com comorbidade psiquiátrica em emergência geral: visão dos enfermeiros. Revista Gaucha de Enfermagem, 40, e20180076. https://doi.org/10.1590/1983-1447.2019.20180076

Portal, P. S. C., Santos, T. de O. C. G., Guimarães, S. do S. de V., Barreiros, M. de P., Pinto, R. B., Dias, C. H., Moraes, P. M. de O., \& Mendonça, X. M. F. D. . (2021). As equipes multidisciplinares como dispositivos "técnicos de referência" em saúde mental nos caps e a gestão do cuidado: uma revisão integrativa de literatura. Research, Society and Development, 10(6), e21010615747. https://doi.org/10.33448/rsd-v10i6.15747

Silva, T. C. S. da, Santos, T. M. Dos, Campelo, I. de G. M. T., Cardoso, M. M. V. N., Silva, A. D., \& Peres, M. A. de A. (2020). Night Admission at a Psychosocial Care Center III. Revista Brasileira de Enfermagem, 73(1), e20170964. https://doi.org/10.1590/0034-7167-2017-0964

Stetler, C. B., Morsi, D., Rucki, S., Broughton, S., Corrigan, B., Fitzgerald, J., Giuliano, K., Havener, P., \& Sheridan, E. A. (1998). Utilization-focused integrative reviews in a nursing service. Applied nursing research : ANR, 11(4), 195-206. https://doi.org/10.1016/s0897-1897(98)80329-7 
Research, Society and Development, v. 10, n. 9, e31010918030, 2021

(CC BY 4.0) | ISSN 2525-3409 | DOI: http://dx.doi.org/10.33448/rsd-v10i9.18030

Veloso, C., Stela, L., Monteiro, D. S., Uchôa, L., Veloso, P., \& Cavalcante, I. C. (2018). Serviço Pré-Hospitalar Móvel De Urgência Psychiatric Nature Care Provided By the Urgent Mobile Pre- Hospital Service. 27(2), 1-10. https://doi.org/10.1590/0104-07072018000170016

Willrich, J. Q., Kantorski, L. P. rad., Antonacci, M. H. ohman., Cortes, J. M. orae., \& Chiavagatti, F. G. opinge. (2014). From violence to bonding: constructing new meanings for attention to the crisis. Revista Brasileira de Enfermagem, 67(1), 97-103. https://doi.org/10.5935/0034-7167.20140013

Willrich, J. Q., Kantorski, L. P., Chiavagatti, F. G., Cortes, J. M., \& Antonacci, M. H. (2013). The meanings constructed in the attention to a crisis in the territory: The Psychosocial Care Center as a protagonist. Revista Da Escola de Enfermagem, 47(3), 657-663. https://doi.org/10.1590/S0080623420130000300019

Yasui, S. (2013). Entre o cárcere e a liberdade: apostas na produção cotidiana de modos diferentes de cuidar. Revista Polis e Psique, 2(3), 5. https://doi.org/10.22456/2238-152x.40317

Zeferino, M. T., Cartana, M. do H. F., Fialho, M. B., Huber, M. Z., \& Bertoncello, K. C. G. (2016). Health workers' perception on crisis care in the Psychosocial Care Network. Escola Anna Nery, 20(3), 1-7. https://doi.org/10.5935/1414-8145.20160059 